\title{
Smart inclusive universities: the role of technology for the accessibility and usability in the Italian context
}

\author{
Luisa Varriale $^{1,}{ }^{*}$, Paola Briganti $^{1}$, and Tiziana Volpe $^{2}$ \\ ${ }^{1}$ University of Naples "Parthenope", Department of Sport Science and Wellness, Italy \\ ${ }^{2}$ ISMED CNR, Italy
}

\begin{abstract}
This paper aims to investigate the Italian universities outlining their degree of accessibility and usability using digital technologies. Considering the crucial role played by the digital technologies in supporting educational and training processes and making them much more accessible and used in practice for students with disability and special needs, we aim to provide a portrait of the Italian universities and their way to be accessible and inclusive adopting the digital technologies (accessible website; visual content in PowerPoint slides; student-facing online tools, such as, clickers, discussion boards, chat bots, and WIKIs accessible, etc.). Starting from a deep review of the literature on the topic, we investigate the Italian universities mapping them considering some specific main features (i.e. the degree of accessibility and usability of website, the adoption of e-learning solutions, etc.). Our qualitative research, through a multiple case study, aims to provide interesting insights for the future analysing one specific country, where some relevant cultural, contextual, geographic and organizational factors can significantly impact the way the universities try to be much more inclusive, adopting digital technologies, and consequently becoming 'smart' universities.
\end{abstract}

\section{Introduction}

Digital technologies, especially ICT, are a fundamental element of the current technological and globalized world. The use of ICT in educational processes has grown considerably during the last two decades, and, especially because of COVID-19 pandemic, making it possible to reach larger sections of the population, including disadvantaged groups. Otherwise, the use of ICT and in general all the digital technologies, is becoming fundamental to make higher education services accessible and usable especially for people with special needs, achieving and fulfilling sustainable development goals (SDGs) (UN Agenda 2030), in terms of infrastructure, industrialization, and innovation (SDG 9), good health and wellbeing (SDG 3), quality and inclusive education (SDG 4). Especially, digital technologies with focus on ICT can be a useful resource to make content more interactive

*Corresponding author: luisa.varriale@uniparthenope.it 
and engaging for learners and to improve the quality of materials. Hence, it is important for teachers to incorporate technology in their teaching to generate benefits for their students, primarily to promote success for traditionally excluded students, such as students with disabilities. Also, it should be important to adopt innovative solutions using technology for making the website of educational institutions accessible and used in practice. Research shows improvements in accessibility and usability for students with disabilities by combining special methods and materials with the use of ICT. Depending on the type of disability that students present, they encounter different barriers to access to higher education, and these can be eliminated by providing alternative forms of learning to students who can learn in different ways and that the teacher must be aware of them. There are various technical aids to incorporate technologies, and each of them is aimed at the accessibility of a certain type of disability (visual, auditory, motor, cognitive).

This paper aims to provide a map of the Italian universities outlining their degree of accessibility and usability in the perspective of social inclusion using digital technologies. Indeed, we investigate the Italian context for giving a picture of the national universities and their way to be accessible and inclusive adopting the digital technologies (accessible website; visual content in PowerPoint slides; student-facing online tools, such as, clickers, discussion boards, chat bots, and WIKIs accessible, etc.). Thus, starting from a deep review of the literature on the topic, we investigate the Italian universities mapping them considering the following main features: the degree of accessibility and usability of their infrastructure through technological solutions, the degree of accessibility and usability of website, the adoption of e-learning solutions, the adoption of e-training solutions, the adoption of assistive technology. Hence, our qualitative research, through a multiple case study, aims to provide interesting insights for the future analyzing one specific country, where some relevant cultural, contextual, geographic and organizational factors can significantly impact the way the universities try to be much more accessible and used in practice, thus inclusive, adopting digital technologies, and consequently becoming 'smart' universities.

\section{Smart and inclusive universities: Theoretical and contextual background}

Over the last thirty years, the academic community has shown a growing interest in issues related to sustainable development, following the spread by organizations of behaviors and actions based on the logic of socio-environmental responsibility and the use of accountability tools that allow stakeholders to be accountable for the commitment of the organizations in pursuing sustainability objectives [1]. At the same time, universities have begun to play a role of agents and subjects of change, though, respectively, the promotion of research and training courses on the subject of economic, social and environmental sustainability, and the definition and internal implementation, above all, of differentiation policies. waste, mobility management (analysis and improvement of home-university-home transport of teachers and students) and inclusion of employees and students with disabilities and special needs [2].

With reference to the functions performed in the economic and social context in which they operate, universities have been called upon to adopt an approach towards sustainability that is different from that of other organizations. The core activities of the universities are represented by teaching, research and development of socially useful links with the territory (Third Mission); hence, the fact that they have found themselves playing an elective role in the process of spreading the logic of sustainability. 
The first official declaration on the commitment that universities have been called to fulfill for sustainability can be traced back to the Talloires declaration, signed in France in 1990: "Universities educate most of the people who develop and manage society's institutions. For this reason, universities bear profound responsibilities to increase the awareness, knowledge, technologies, and tools to create an environmentally sustainable future. Universities have all the expertise necessary to develop the intellectual and conceptual framework to achieve this goal. Universities must play a strong role in the education, research, policy development, information exchange, and community outreach to help create an equitable and sustainable future" [3].

The role of universities for sustainability was further strengthened by the UN Agenda 2030, adopted in 2015 by the General Assembly of the United Nations, consisting of 17 Sustainable Development Goals (SDGs), framed within an action program consisting of 169 targets or goals, associated with them, to be achieved in the environmental, economic, social and institutional pillars by 2030. Through the 17 SDGs, the 2030 Agenda aims to address the challenges in a coordinated and shared manner most urgent on the planet, including ending poverty and bringing economic prosperity, social inclusion and environmental sustainability. Universities are called upon to lead the construction of that "transformative resilience" necessary for the concrete implementation of the 17 SDGs [1]. The invitation to follow specific operational practices to best fulfill the role for sustainability was represented by the implementation plan of the Manifesto "From 'Universities for sustainability' to 'Sustainability in Universities'", drawn up by the University Network for Sustainable Development (Rete delle Università per lo Sviluppo Sostenibile - RUS) and signed by the CRUI in May 2019 in Udine, which identifies concrete actions to be implemented along different operational lines.

The Manifesto is configured as a "pact" between the Rectors of Italian universities, not regulated by laws, but of pregnant ethical value, which commits Italian universities to promote the implementation of the 2030 Agenda, in order to strengthen the role of universities in the socio-economic transformation process of the country, to support the sustainable development of local and territorial communities.

In this direction, universities have been called upon to contribute to the maturation of awareness of sustainability in the dual role of "agents of change" and "subjects of change" [1].

One of the most important and delicate issues that requires a reactive and, in the medium-long term, proactive role of universities is represented by social sustainability and, in particular, by social inclusion, to be promoted and implemented above all through the Third Mission, taking an interest in developing innovative services and solutions in the housing, family and assistance and care sectors for the most needed social groups (fragile categories), often invisible to the eyes of local institutions and silently left on the margins of communities.

Several existing phenomena, such as the increase in the demographic age of the population, the high rates of unemployment and drop-outs and inactivity especially among young people, and the growing influx of immigrants, make traditional corporate and social welfare policies absolutely inadequate in terms of timely and useful responses to be given to a whole spectrum of emerging social needs and new inequalities.

Universities have been called to follow a multi-stakeholder public engagement approach, to implement projects that strengthen links with the territory and the civil and economic community of reference, acting as levers of an institutional direction virtuously connected to the themes of sustainable development [2]. 
Therefore, it is interesting to map the state of the art in terms of education for sustainable development in Italian universities, a complex and delicate operation, as there are not currently objective criteria for identifying and classifying the many activities falling within the category of education for Sustainable Development. Several studies have found it sufficient that the title of the university's activity contain a more or less explicit reference to the theme of sustainability, a percentage of minimum hours or study materials within the training program dedicated to the theme of sustainable development.

Furthermore, mapping turns out to be a challenging operation with heterogeneous and nuanced margins because sustainable development is a transversal issue that can be treated by all scientific disciplinary sectors, following different approaches and different intensities of planning and action. Finally, the picture is constantly evolving and rapidly developing.

The WG RUS - Education has classified the practices on the subject of the SDGs included in the RUS universities into six categories [2]:

a) Lesson Zero, or transversal training module aimed at basic literacy. b) Bachelor's, three-year or master's degree courses. c) Higher Education Courses (doctorates, summer school, business school, masters). d) Curricular modules $\backslash$ activities on the theme of sustainable development, in non-specific training courses. e) Training for teaching and administrative staff; f) Extra-curricular activities, non-formal and informal activities for students, of an experiential type.

Defining useful boundaries for mapping the various activities is necessary and urgent. An initiative to support the dissemination of CSR teaching within training courses is the subject of the United Nations and called "United Nations' Principles for Responsible Management Education" (PRME), which aims to promote the pursuit of sustainable development goals (SDGs) by the member countries through the preparation of socially responsible managerial training courses $[4,5]$. The data provided by the United Nations show how the implementation of this program within the member states has favored the implementation of over 660 university courses with about 20 million students involved in it (United Nations, 2017). This intervention also concerns Italian universities, as indicated in the PRME which identifies, in 2018, a total of 8 Italian universities that have joined the training program. It should be remembered that over the last decade, from the didactic aspect, CSR has been gradually accepted within the training courses of Italian universities [6].

The purpose of this research is, therefore, to evaluate the current degree of openness towards these issues by the Italian university system by providing an analysis of the Italian university realities considering two different guidelines: on the one hand, focus on the internal organization of universities highlighting existing strategies and policies and consequent behavioral models in a perspective of global inclusion and wide-ranging sustainability; on the other hand, focus on proposals and interventions in terms of training, research and Third Mission activities implemented by universities in the perspective of inclusion and sustainability involving the entire community.

Moreover, in an age of mobility and diffusion of digital technologies, such as mobile phones and applications, another significant topic of this research about universities and inclusion is represented by the role of technology, including mobile learnings. During the last 15 years, learning with mobiles have been developed in UK, Western Europe, US and Asia Pacific, integrating the activity in universities, if students can bring their own device. Continued and dramatic changes in the ownership, access and expectations of mobiles among university students and across the world have suddenly transformed learning with mobiles in a primary tool of learning and access to university services, for the minorities too. Discussion about authority, agency and control within university settings about the 
continued and dramatic changes in the ownership, access and expectations of mobiles among university students and around the world, and the equally significant and profound implications for the learning and inclusion have not still been well articulated and analysed in universities and policy makers agenda. To begin that process, Traxler [7] identified and discussed a theoretical framework for social inclusion in the university through mobiles. The Author [7] reviewed the progress and problems of the substantial and unique programme of mobile learning across UK higher education since 2000 in relation to inclusion, to understand if mobile learnings contribute only apparently to perform in an including way for respecting social and institutional pressures, to understand programme's meaning and direction in a world where now there is more, better, cheaper, faster, newer but different digital technology in the hands of students, potential students and everyone else than there is routinely in the educational institutions themselves. This digital technology, especially mobile technology, now allows learners to create, own, transform, discuss, discard, share, store and broadcast ideas, opinions, images and information, and to create and transform identities and communities. This epistemological revolution may mean that universities and colleges are no longer credible and authoritative gatekeepers to knowledge and its technologies and so the meaning and relevance of inclusion are much less clear. We need to develop a new stance on inclusion.

For instance, Pira [8] studied how to promote the social inclusion of students with autism spectrum disorder via mobile technologies. Autism Spectrum Disorder (ASD) is the most commonly diagnosed neurodevelopment disorder in the world. Students with autism demonstrate impairments in language, communication skills, and social interactions: they show serious difficulties to communicate and interact with their peers, and educators. During last 10 years, many interventions were based on the use of mobile technologies in assisting students with ASD in developing their social, communication, language, and other educational skills required for their academic success, despite social inclusion, and learning progress of these students in the classrooms is still challenging. Pira [8] explored how students with ASD are socially included in the learning activities using mobile technologies and the impact on educators and parents in Canada. Educators, other education professionals, and parents participated in surveys and one-on-one interviews to provide further insights about their experiences in meeting students' communication, social, language, and educational needs via mobile technologies. "Analysis of both quantitative and qualitative data resulted into four emerging themes: (a) a balanced learning model where social inclusion of students with ASD is supported through the balance between mobile technologies and personal interaction; (b) resources and supports such as open educational resources, funding to support mobile technologies, evidence-based knowledge on mobile technologies, and training for all stakeholders are necessary in creating a socially inclusive environment for students with ASD; (c) goal oriented and needs-based usage where mobile technologies are used for addressing the needs of students with ASD and for achieving a particular goal or purpose in education; and (d) a team oriented approach that involves collaboration among students, parents, school administrators, educators, other education professionals, and various stakeholders in promoting the social inclusion via mobile technologies. The findings from this research study indicated various aspects (promoters) that are important in promoting the social inclusion of students with ASD via mobile technologies. Through these promoters, effective levels of social inclusion can be achieved where students' communication, social, language, and educational needs are addressed sufficiently" [8].

Another critical topic is to develop efficacy and equity methods to evaluate the accessibility and usability of the university environment, especially the virtual environment, assessing for instance the effectiveness and usefulness of online learning materials for students with disabilities, such as visual or hearing impairments. Previous studies show that 
university websites have become gradually more inaccessible for students with disabilities. Some scholars [9] sampled a random selection of 20 universities from each of the Academic Ranking of World Universities top 100, Oceania region top 50, and Arab region top 50 ranked universities. They evaluated three types of website from each university (home page, the admission page, and a course description page), revealing 30,944 accessibility related home-page errors among the 180 evaluated webpages. Comparison with earlier studies reveals no significant improvement in the accessibility and usability of university websites between 2005 and 2015 . There were also no differences in accessibility levels amongst the selected top-ranking universities in the world. Therefore, there is a growing need for universities to improve accessibility of online learning materials for students with disabilities.

Besides technology for students with learning special needs, the web represents, in general, a significant part of higher education and let students to get an unprecedented level of access to information and services: there is a growing need of universities and colleges to make the necessary adjustments to ensure the accessibility and usability of their websites' content. During the last two decades, there has been an important development of technical standards and accessibility laws and studies about Web content accessibility, which remains a concern in higher education. Laufer and Rimmerman [10] analysed mandatory Web accessibility requirements applicable to higher education in Israel, during a period of evolving legal changes in this way.

"An automated evaluation tool was used to measure the adherence of the sample Web pages to the technical standards, and results showed that all examined Web pages presented accessibility barriers and were non-compliant with the most basic conformance level. 'Contrast' and 'missing alternative text' errors were the most frequent problems identified in the evaluation. The library's Web pages exhibit relatively better level of accessibility compared to the other examined Web pages of the university. The research highlights the need for clear and enforceable legislation to encourage academic Web accessibility. Additionally, technical training and awareness raising could be key elements in improving accessibility" [10].

Another study [11] tested if universities websites were accessed effectively, efficiently and satisfactorily by the whole target group of users, in the top universities in Kyrgyzstan, Kazakhstan, Azerbaijan and Turkey, adopting automated assessment tools. The results demonstrated that Turkey and Turkish universities developers pay more attention to the performance of websites, followed by Azerbaijani, Kyrgyz and Kazakh universities. According to the World Wide Web Consortium (W3C), that provided the most important set of guidelines for web accessibility (WCAG), the majority of the university websites in the study didn't meet the basic (Web Content Accessibility Guidelines - WCAG 2.0) accessibility standards criteria, highlighting the need to devote more effort to make their websites more accessible and used in practice for their users.

A further study [12] analysed accessibility of higher education college websites affiliated with the Indian University of Kashmir and Cluster University Srinagar. The Authors used two significant accessibility evaluation tools called web accessibility test (TAW), and the accessibility engine powering browser extensions (accessibility engine aXe), and they list the major accessibility barriers exposed by these sites in terms of metrics, and a number of problems, warnings, status of success criteria violations. Findings of this statistical analysis represent a driver for making these websites inclusive and barrier-free.

A critical specific issue is represented by libraries accessibility of websites and electronic resources by individuals with and without mental, physical or other impairments, Kimura [13] analysed digital accessibility in libraries, and, in particular, practical guides for authors of web content, principles of universal design, ethical considerations of libraries 
subscribing to packages of digital content, critical examinations of the accessibility guidelines themselves, considering that many tools have become quickly outdated, and more attention should be paid to technologies' change on the matter of learning digital resources.

This paper focuses on the way universities through digital technologies try to be much more accessible and used in practice, becoming smart and inclusive. In this direction, the idea is to provide to universities and all the stakeholders useful information about new challenges that universities have to accomplish with, considering the significant role of websites and learning inclusiveness, in general, and for people with disability and special needs, assisting universities in planning and implementing effective solutions for being much more "smart and inclusive" considering the increasingly complex scenarios.

\section{Methodology}

Starting from a review of the main contributions of the literature and practice and adopting a qualitative methodology, this study provides an in-depth analysis of the technological solutions and initiatives introduced in the Italian universities in the perspective of social inclusion.

This empirical analysis aims to provide a portrait of Italian universities, state and nonstate, deepening the behavioural models and initiatives introduced from the perspective of social inclusion using digital technologies and, thus, outlining the degree of inclusiveness related to technological innovativeness. In detail, this study adopts a multiple case study approach $[14,15]$, a method that is usually used to achieve descriptive purposes. The research, in fact, was mainly conducted to describe the current state of the degree of inclusiveness related to technological innovativeness of Italian universities. Most scholars say that a qualitative study cannot validate theoretical hypotheses or move towards a "grounded theory", because it represents a case study [16]. In our qualitative study we follow a specific iterative logical methodology (Figure 1), with a selection of cases made by applying specific criteria. The sample is made up of 85 case studies of universities throughout the country that present a different degree of inclusiveness related to technological innovativeness.

The sample of Italian universities was created using the Universitaly website, the portal of the Ministry of University and Research (MUR), an online platform that allows you to collect all useful information about the universities operating in Italy with its offer, training and referrals through links available to official websites and all their existing communication channels. The portal provides a summary sheet for each university highlighting its size (mega, large, medium and small), nature (state, non-state, telematic) and geographical area of reference (North, South, Center, Islands).

The sampling was, therefore, carried out through the Universitaly portal, selecting only universities that met the selected criteria, i.e. "presence in the Universitaly portal, ANVURMUR accreditation, and website presence". 


\section{PHASE 1}

REVIEW: Main contributions in research and practice (studies and international reports and contributions focused on the Italian context) on universities and their technological and innovative solutions in terms of promotion and development of inclusion and sustainability through, i.e. in terms of adoption and dissemination of initiatives, interventions and policies (also for the training offer) using digital technologies, with a view to inclusion and sustainability for the definition of research questions.

RESEARCH QUESTIONS: What are the strategies, policies and initiatives developed and introduced by Italian universities in order to be "inclusive" and sustainable through the adoption of digital technologies? How do Italian universities deal with the phenomenon of social inclusion through digital technologies in their organization? What is the degree of inclusiveness of Italian universities using technology?

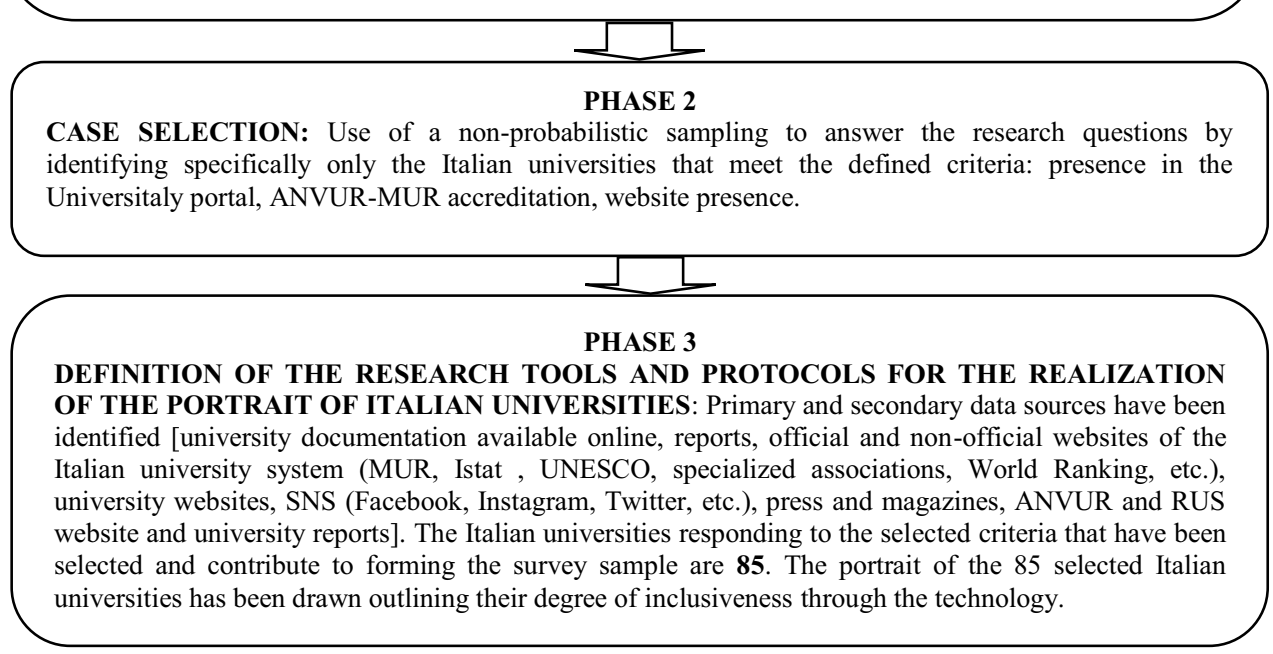

We conducted a manual content analysis of all the documentation, precisely primary and secondary data were taken into consideration. The framework of the strategies, policies and initiatives promoted and implemented by the investigated Italian universities was built by collecting and processing information and data through reports (MUR, Istat, etc.), analysis of archival data, university documentation, university websites, university social network sites (SNS), press and magazines, focusing on issues related to inclusion and digital technologies.

We have identified and investigated all the solutions, policies and initiatives promoted and introduced by the universities analysed to outline their overall degree of inclusiveness linked to the digital technologies.

To complete the analysis, some of the most significant initiatives and experiences carried out by some small, medium, large and mega-sized universities were compared to identify the key organizational and managerial implications that characterize the main differences with a specific focus on their approach and orientation to inclusion and technological innovativeness. Consequently, we have built a portrait of Italian universities as an inclusive environment because accessible and used in practice university environments through the adoption of digital technologies. 


\section{Results and Discussion}

The sample analysed consists of 85 universities of which 59 state universities and 15 non-state universities, 11 are telematic universities and there is 1 university for foreigners. Among the 85 universities, only 49 universities have a specific figure, such as delegate, pro-rector or advisor to the Rector, who is entrusted with the function linked to the area of disability and social inclusion. In the selected sample, 16 universities are not registered in the sustainability network (RUS), of which 3 state, 10 telematic and 3 non-state, and these are mainly small and medium-sized universities.

inclusion.

Only 10 universities provide website with the acoustic reading support for blind-visually impaired people, where only 1 as public university.

Our analysis shows that universities investigated do not have any support in their website in terms of choosing colours according to the specific regulation and major guidelines for visually impaired people.

In the sample 68 universities promote and adopt technological solutions for students with special needs (e.g. Interactive Multimedia Whiteboard - Lavagna Interattiva Multimediale LIM; acoustic reader; audiobook; computer with touchscreen). Among these universities we can distinguish 12 non-state universities, 48 state, and 8 telematic universities and they are mostly large and mega universities (19) in the north and centre of the country.

Also, 65 universities provide video lessons, short videos, multimedia material created ad hoc. Most universities that adopt technological solutions generally also offer multimedia materials, among them 30 universities provide online support, primarily state universities (27), and 24 universities provide technical support. Thus, we can observe that most often universities which provide technical support also consider online support.

Finally, 78 universities provide online courses, thus, only 8 universities do not have online courses, 5 of which are non-state courses and have small size. There are 6 universities which offer the possibility to download apps, 5 have digital assistants, 4 of which are nonstate, and 1 university has a dedicated call centre and 1 toll-free number.

The main lines of activities promoted and implemented by universities with a view to disability and inclusion, with concern of the degree of accessibility and usability in practice of the physical and virtual environment, can be traced back to five areas of competence: 1) support for vulnerable groups aimed at students with disabilities, SLD and/or BES; 2) reception and accommodation desk, aimed at non-resident students, researchers and teachers who are guests of the University; 3) psychological counselling aimed at students and structured staff (teaching and non-teaching); 4) internal mobility and architectural barriers; 5) definition and implementation of IT support tools. In these five areas we can observe several technological solutions implemented in order to promote social inclusion making the universities much more inclusive.

Most universities have accommodations without architectural barriers and equipped for various disabilities, such as the University of Trento in the 'San Bartolomeo' student residence and in the apartments in the centre, also with possible solutions to accommodate a family member or a trustworthy person for assistance needs. "There are physical-structural accessibility maps in the departments/centres. The University of Trento promotes participation in national and international networks, aimed at developing policies on disability, with particular reference to university contexts, including the National University Conference of Delegates for Disability (CNUDD), established since 2001 at the Conference of Rectors of Italian Universities (CRUI), the Coordination of the Triveneto Universities for Inclusion (Uni3V), which saw the University of Trento as one of the promoters of the 
National Conference for Equality Bodies of Italian Universities, the RUS, and in particular the Working Group 'Inclusion and social justice' "(www.unitn.it).

A "dissemination service of information relating to the accessibility of the offices has been activated at the University of Udine with the possible provision of illustrated guides. In addition, a transport service is offered for travel between universities (including the Student House) located within the municipalities of Udine and Gorizia, especially for students with disabilities. The following services are also active using mostly advanced technologies: the self-study support service for visually impaired students; the loan of portable PCs with the installation, upon request, of video-reading programs and for the retrieval of texts at the University libraries; assistance service, in collaboration with the competent structures involved from time to time, for the evasion of bureaucratic procedures; the use of an optikbook scanner, a Topaz video-enlarger and a Maestro station at the headquarters; a specific guidance service aimed at choosing the course of study" (www.uniud.it).

In general, with regard to policies on disability in its various dimensions and inclusion, most universities tend to introduce classic compensatory measures, such as the service charter, exam support, counselling and psychological listening services, the digitalization of texts, specialized peer tutoring, total accessibility to buildings and classrooms, the definition of differentiated programs, the recognition of concessions for university fees and the design of personalized interventions. Thus, most interventions tend to adopt technologies for promoting the inclusion and integration of students with disability or special needs.

In 2020, the University of Tuscia purchased the license for the Easyreading font, which "provides a font dedicated to those with dyslexia. It is a highly readable typeface that overcomes reading barriers even for those with dyslexia. It is used as a default for carrying out telematic educational activities. The aim is to facilitate the academic path of students with SLD, offering them a new type of teaching outside the classical schemes and closer to their needs. The University has the right to use the Font for installation on computers within its own facilities and on the personal ones of teachers in order to create, both in paper and digital format, didactic material, printed matter, non-commercial publications and facilitate internal and external communication, including for the University website" (www.unitus.it).

In other cases, such as at the University of Foggia, a series of services is offered: "an au pair tutoring activity, different solutions such as additional time up to a maximum of $30 \%$ more, the opportunity to carry out checks oral rather than written, also taking into account the individual skill profile, the evaluation of the contents rather than the form and spelling, and solution technologically supported like the recording of the lessons, the use of texts in digital format and speech synthesis programs" (www.unifg .it).

In addition, the Lombard universities participate and share their experience with the CALD, the coordination network of the Lombard universities for disability that the Rectors' Delegates established in 2011 to more effectively implement university legislation in favor of the full inclusion and participation of students with disability and SLD to university life.

In most cases, in order to promote equal opportunities, Italian universities present within themselves a Single Guarantee Committee (CUG) which, in particular, aims to support the integration of gender equality in all the policies of University, through tools such as the Gender Report and the Positive Action Plan, or the action aimed at promoting the experimentation of space-time ways of carrying out "innovative" work performance (for example, smart working), in coherence with the requests aimed at facilitating the harmonization of professional life with private life that emerge from the legal framework of 
reference, as well as from the organizational sciences, and which have seen their strategic role grow rapidly, and not without criticality, with the explosion of the epidemiological emergency from Covid-19. To this end, the intervention of the CUG becomes even more effective through the figure of the guarantors of behaviour. In particular, these guarantors promote and implement the following services and initiatives: reception services to facilitate the university career of the weakest and most difficult categories; initiatives to favour the chosen training path; assistance services, technological aids, didactic and specialized tutoring; public selections for part-time contracts with "senior" students; initiatives to improve the working conditions of disabled staff; the study of forms of activities aimed at improving the working conditions of staff with dependent family members with disabilities; monitoring of architectural barriers; relations with the CUG.

The analysis conducted which briefly outlined a summary framework of Italian universities regarding the topic of disability and special needs allows for some considerations specifically about the degree of accessibility and usability in practice of the same universities through the use and adoption of digital technologies (see Table 1 for a summary):

1. the majority of universities that have policies and measures aimed at promoting inclusion making their environment physically and virtually (website) accessible and usable in practice tend to substantially transpose and apply the current legislation on the protection of protected categories or guarantees for the fragile or in difficulty categories;

2. the universities that have a greater orientation towards inclusion are concentrated in Northern and Central Italy, feeding the historical geographical gap existing in the country;

3. mega, large and medium-sized universities stand out more for the promotion and adoption of technological solutions for being much more inclusive;

4. the universities most sensitive to inclusion issues tend mainly to promote and introduce blended or online learning courses, virtual supportive tools or digital assistance, and specific programs using digital technologies for students with disability or special needs.

Table 1: A brief portrait of the Italian Universities.

\begin{tabular}{|c|c|c|c|c|c|c|c|}
\hline $\begin{array}{l}\text { SAMPLE OF UNIVERSITIES } \\
\text { DISTINGUISHED IN TERMS } \\
\text { OF } \\
\text { SIZE/NATURE/GEOGRAPHI } \\
\text { C AREA }\end{array}$ & $\begin{array}{c}\text { RUS } \\
\text { MEMBER }\end{array}$ & $\begin{array}{l}\text { PRESENCE OF } \\
\text { PROTECTED } \\
\text { CATEGORIES } \\
\text { POLICIES }\end{array}$ & $\begin{array}{l}\text { PRESENCE OF } \\
\text { DISABILITY } \\
\text { MANAGEMENT } \\
\text { POLICIES }\end{array}$ & $\begin{array}{c}\text { DIGITAL } \\
\text { ASSISTANTS }\end{array}$ & $\begin{array}{c}\text { CALL } \\
\text { CENTER }\end{array}$ & $\begin{array}{l}\text { TECHNICAL } \\
\text { \& ONLINE } \\
\text { SUPPORT }\end{array}$ & $\begin{array}{c}\text { ONLINE } \\
\text { COURSES } \\
\text { (E-LEARNING } \\
\text { PLATFORM) } \\
\end{array}$ \\
\hline Mega Universities & 10 & 7 & 10 & 2 & 11 & 20 & 20 \\
\hline Large Universities & 20 & 15 & 20 & & 4 & 10 & 10 \\
\hline Medium Universities & 21 & 10 & 22 & & 7 & 21 & 21 \\
\hline Small Universities & 13 & 13 & 17 & 2 & 4 & 13 & 13 \\
\hline State Universities & 55 & 37 & 49 & 2 & 23 & 54 & 54 \\
\hline Non-state Universities & 12 & 9 & 14 & 3 & 4 & 12 & 12 \\
\hline Telematic Universities & 1 & 5 & 6 & & 3 & 11 & 11 \\
\hline North Universities & 25 & 21 & 26 & 2 & 9 & 24 & 24 \\
\hline Centre Universities & 27 & 19 & 30 & 3 & 12 & 32 & 32 \\
\hline South Universities & 11 & 9 & 17 & & 5 & 17 & 17 \\
\hline Islands Universities & 5 & 3 & 5 & & 4 & 5 & 5 \\
\hline
\end{tabular}




\section{Concluding remarks}

This study provides a synthetic analysis of the Italian context by taking a picture of the Italian universities from the perspective of inclusion and technological innovativeness. In particular, this study underlines the need to create the conditions to activate an open comparison between Italian universities in order to share experiences and good practices in order to create inclusive and sustainable organizations using advanced technologies for supporting all people, especially students, to easily and directly access and use in practice the services and all the structural and infrastructural tools available. Indeed, we observe that there is a geographical and dimensional gap between universities in terms of the adoption of digital technologies for being much more accessible and used in practice, thus inclusive. Also, there is the need to internally promote at universities, recognized as exclusive managers of the training of future generations, a process of radical change in the individual and organizational culture to truly acquire the fundamental principles of inclusion, so as to continue this process of change externally involving the entire community, in order to allow universities to become "smart and inclusive".

Starting from the limitations of this study, considering its exploratory nature, in the future we plan to directly involve the viewpoint of students with disabilities and/or special needs conducting in-depth semi-structured interviews about technological solutions provided from their university, and also to investigate directly some specific universities in order to collect useful information and data about the concrete degree of accessibility and usability of the environment of universities in the perspective of becoming 'smart and inclusive'.

The study highlights that university environments can be suitable for the education, in particular for students with intellectual disability; therefore, the creation of inclusive higher education programmes should be encouraged, through training the same University teachers to be much more inclusive (referring to the critical issues facing the education system today, that is the lack of highly qualified and well trained professionals who are capable of working with the increasing numbers of students with specific needs referred for special education supports and services), and through Higher education institutions that must take into account the voices of vulnerable groups of students in order to effectively eliminate the existing barriers.

From a managerial perspective, inclusive Universities should create educational and administrative guidelines for strengthening inclusiveness in the university programmes for both students with intellectual impairments and the overall university community [17],

More inclusive universities services and special educators will be needed in the next years to come: there are high challenges to face that is to find high quality potential special educators from a variety of backgrounds and experiences, able to program and manage inclusive services; there is the need of special educators equipped with more effective teaching strategies for a very diversified student population and higher educational inclusive tools; the engagement in an international pursuit requires to retain and improve the teacher efficacy and quality in inclusive settings given a changing educational policy context [18].

\section{References}

1. RUS \& Gruppo di Studio per il Bilancio Sociale. Il Bilancio di Sostenibilità delle 
Università (2021)

2. CRUI. I Magnifici Incontri Crui 2019 'Le Università Per La Sostenibilità - Educazione Universitaria Per La Sostenibilità' (Position paper) (2019)

3. https://www.google.it/url? sa=t\&rct=j\&q=\&esrc=s\&source=web\&cd=\&cad=rja\&uact $=$ 8\&ved=2ahUKEwixobnz9jzAhUAhf0HHeSoBFYQFnoECAMQAw\&url=https $\% 3 \mathrm{~A} \%$ 2F\%2Fulsf.org\%2Frepo rt-and-declaration-of-the-presidents-conference1990\%2F\&usg=AOvVaw15sOAGy_xfb1Si8y-0TjKk (Report and Declaration of The Presidents Conference, 1990)

4. A. V. P. F. Caputo, S. Pizzi. L'offerta di contenuti di responsabilità sociale negli atenei italiani. Un'analisi esplorativa (2019)

5. J. Godemann, J. Haertle, C. Herzig, J. Moon. 'United Nations supported principles for responsible management education: purpose, progress and prospects', in Journal of Cleaner Production; 62: 16-23 (2014)

6. C. Gulluscio, P. Torrecchia, T. De Cristofaro. CSR Modules in Catholic and Ecclesiastical Universities in the Italian Territory. In Key Initiatives in Corporate Social Responsibility (pp. 131- 156). Springer, Cham (2016)

7. J. Traxler. Inclusion in an age of mobility. Research in learning technology; 24 (2016)

8. R. Pira. Promoting the Social Inclusion of Students with Autism Spectrum Disorder via Mobile Technologies (Unpublished doctoral thesis). University of Calgary, Calgary, AB. Uri: http://hdl.handle.net/1880/114036 (2021)

9. T. Alahmadi, S. Drew. Accessibility evaluation of top-ranking university websites in world, Oceania, and Arab categories for home, admission, and course description webpages. Journal of Open, Flexible and Distance Learning, 21(1): 7-24 (2017)

10. H. L. Nir, A. Rimmerman. Evaluation of Web content accessibility in an Israeli institution of higher education. Universal Access in the Information Society, 17(3): 663-673 (2018)

11. R. Ismailova, Y. Inal. Accessibility evaluation of top university websites: a comparative study of Kyrgyzstan, Azerbaijan, Kazakhstan and Turkey. Universal Access in the Information Society, 17(2): 437-445 (2018)

12. A. Ismail, K. S. Kuppusamy. Web accessibility investigation and identification of major issues of higher education websites with statistical measures: A case study of college websites. Journal of King Saud University-Computer and Information Sciences (2019)

13. A. K. Kimura. Defining, evaluating, and achieving accessible library resources: A review of theories and methods. Reference Services Review (2018)

14. R. K. Yin. Qualitative research from start to finish. New York, NY, USA: The Guilford Press (2015)

15. B. Yazan. Three approaches to case study methods in education: Yin, Merriam, and Stake. The Qualitative Report, 20(2), 134-152. (2015) 
16. B.G. Glaser, A.L. Strauss. Discovery of grounded theory: Strategies for qualitative research. Routledge. doi: 10.4324/9780203793206. (2017)

17. M. P. Calderón Albornoz, P. Rodríguez Herrero, P. Social representations of intellectual impairments in universities: the educational implications of a case study in Chile. Disability \& Society, 1-18. (2021)

18. M. A. Fallon. "11. PREPARING INCLUSIVE SPECIAL EDUCATORS: POLICY IMPLICATIONS FOR PARTNERSHIPS AMONG PUBLIC SCHOOLS, COLLEGES, AND UNIVERSITIES", Alexander, K.and Hunter, R.C. (Ed.) Administering Special Education: In Pursuit of Dignity and Autonomy(Advances in Educational Administration, Vol. 7), Emerald Group Publishing Limited, Bingley, pp. 239-248. https://doi.org/10.1016/S14793660(04)07011-8. (2004) 